\title{
PARAMETER IDENTIFICATION AND ESTIMATION FOR STAGE-STRUCTURED POPULATION MODELS
}

\author{
CARMEn COLL ${ }^{a}$, Elena SÁNCHEZ ${ }^{a, *}$ \\ ${ }^{a}$ Institute of Multidisciplinary Mathematics \\ Polytechnic University of Valencia (UPV), Camino de Vera, 14, 46022 Valencia, Spain \\ e-mail: esanchezj@mat.upv.es
}

\begin{abstract}
A stage-structured population model with unknown parameters is considered. Our purpose is to study the identifiability of the model and to develop a parameter estimation procedure. First, we analyze whether the parameter vector can or cannot uniquely be determined with the knowledge of the input-output behavior of the model. Second, we analyze how the information in the experimental data is translated into the parameters of the model. Furthermore, we propose a process to improve the recursive values of the parameters when successive observation data are considered. The structure of the state matrix leads to an analysis of the inverse of a sum of rank-one matrices.
\end{abstract}

Keywords: system identification, parameter estimation, dynamic population, discrete-time system, rank-one matrix.

\section{Introduction and mathematical background}

Stage-structured models are based on the assumption that a large group can be divided into smaller ones with similar parameters and in some way interconnected with one another. For example, the individuals of a population are organized in stages because of their evolution in different phases from birth to death. Although Leslie's model is quite a suitable approach for some type of animals, it does not generally conform to the analysis of the evolution of a population of many species. This is the case for some animals such as insects, turtles and even some mammals (Carnia et al., 2015), or when analyzing evolving populations of most plants. Detecting whether a plant will prosper or not depends more on the size or condition of the plant than on its age. This means that those plants that do not reach the desired size in an interval of time will remain in the same class and will not advance to the next one. This behavior does not fit into a Leslie model and in these cases the Lefkovitch model fits better since the elements of the state matrix represent the transition rate from one class of a stage (or size) to another.

When populations are structured, the birth rate is often called fertility (the number of offspring that

*Corresponding author are produced per female of a given stage). Usually, this fertility is zero for an individual in a pre- or post-productive age and positive fertility is observed for individuals of a reproductive age. These structured systems are employed to model the changes in a population of organisms and are widely used in studies of biology, ecology and demography to determine the growth of a population, (see, e.g., Caswell, 2001; Kajin et al., 2012; Leslie, 1948; Lefkovitch, 1965). Note that this kind of dynamic process is represented by a discrete linear system with nonnegative states and nonnegative inputs. Therefore, we are considering positive systems and the study involves the use of nonnegative matrix theory and positive control theory (Kaczorek, 2002). These mathematical tools are usually used in the analysis of the evolution of several real processes (see, e.g., Cantó et al., 2014; Cao and Zhou, 2012; De La Sen and Quesada, 2003; Emmert and Allen, 2004; Li and Schneider, 2002; Li and Wang, 2006).

The notation and the basic concepts that will be used throughout the work are as follows (see Berman and Plemmons, 1994). A matrix $M=\left(m_{i j}\right)$ is nonnegative if $m_{i j} \geq 0$ for all $i, j$, and it is denoted by $M \geq 0$. Analogously, a nonnegative vector is defined and denoted by $\mathbf{p}=\left(p_{i}\right) \geq 0$ if $p_{i} \geq 0$, for all $i$. From now on, we will denote by $e_{i}, i=1, \ldots, n$ the unit canonical basis of $\mathbb{R}^{n}$ and by $\operatorname{col}\left(X_{i}\right)_{i=1}^{l}$ a column matrix by blocks whose 
blocks are $X_{i}, i=1, \ldots, l$.

Our mathematical model considers female populations in each stage as a nonnegative state variables. Sometimes the process is altered by a nonnegative entry of a new population. For instance, an inflow of elements into the first stage appears modifying the dynamic of the population (Caswell, 2001). In this way, we have the dynamics of the process represented by the discrete linear system

$$
\bar{x}(t+1)=A \bar{x}(t)+B, \quad t \geq 0,
$$

where $\bar{x}(t) \in \mathbb{R}_{+}^{n}$ represents the female population and the measure of a new population is given by $B=b e_{1}$; that is, there exists only the entry of a new population at the first stage. In this model, the individuals are organized in $n$ stage classes. Class $j$ includes the female individuals of this stage, and, at time step $t$, some individuals are going from class $j$ to $j+1$ but some of them remain in class $j$. Many species have different stages, such as eggs, juveniles or larvae, adults, etc. Then, the population distribution entails that not all individuals reach the next stage at the same time. In this status, we have the Lefkovitch model given by

$$
A=\left(\begin{array}{ccccc}
\tau_{1}+f_{1} & f_{2} & \cdots & f_{n-1} & f_{n} \\
s_{1} & \tau_{2} & \cdots & 0 & 0 \\
\vdots & \ddots & \ddots & \vdots & \vdots \\
\vdots & \ddots & \ddots & \tau_{n-1} & \vdots \\
0 & 0 & \cdots & s_{n-1} & \tau_{n}
\end{array}\right),
$$

where the entries $f_{j} \geq 0$ are the fertility parameters, at least one of them nonzero, $0<s_{j} \leq 1$ is the survival rate growing into the next stage, and $0 \leq \tau_{j}<1$ the survival rate remaining in the same stage. It is clear that in these models the fertility in the first stage is usually zero. We will assume that $f_{1}=0$. From this matrix, it is possible to calculate a stable age distribution. Note that the biological interpretation of the parameters leads to a system with nonnegative matrices, $A \geq 0$ and $B \geq 0$. Hence, the system is positive (see Kaczorek, 2002).

On the other hand, if the models involve unknown parameters, it is essential to obtain their estimated values to predict or control the behavior of the real process.

We denote by $S(\mathbf{p})$ the class of stage-structured discrete-time linear systems $x(t+1)=A(\mathbf{p}) x(t)+B$ with the parameter vector $\mathbf{p}$ belonging to a suitable subset $\mathcal{P} \subseteq \mathbb{R}^{l}$, and by $i o(\cdot)$ the input-output response obtained from an initial condition and an input. Given a system in $S(\mathbf{p})$, we will use its Markov parameters $V(j, \mathbf{p})=$ $A^{j}(\mathbf{p}) B, j \geq 0$ to determine the input-output behavior of this system.

We say that $S(\mathbf{p})$ is globally identifiable if and only if, for any two candidate parameter vector values $\mathbf{p}, \mathbf{q} \in$ $\mathcal{P}$, the same input-output behavior $i o(\mathbf{p})=i o(\mathbf{q})$ implies $\mathbf{p}=\mathbf{q}$.
Thus, while the identifiability of the model guarantees that the parameter vector can uniquely be determined by the knowledge of the input-output behavior of the model, the estimation responds to how the information in the experimental data is translated into the model parameters. The identifiability guarantees that the model parameters can be estimated under ideal conditions. Usually, the solution of the estimation problem is obtained through minimization of a least squares function. Accordingly, the estimation problem is usually addressed by fitting model simulations to the observed experimental data set $\left\{o b(i) \in \mathbb{R}^{n}, i=\right.$ $1, \ldots, K\}$. To solve this problem, the system $x(t+1)=$ $A(\mathbf{p}) x(t)+B$ is rewritten as

$$
x(t+1)=M(t) \mathbf{p}+N(t), \quad t \geq 0,
$$

and the objective is to minimize the quadratic cost function

$$
J_{K}(\mathbf{p})=\frac{1}{2} \sum_{i=1}^{K} e(i)^{T} e(i)=\frac{1}{2} \mathbf{e}_{K}^{T} \mathbf{e}_{K},
$$

where $e(i)=(o b(i)-x(i)), i=1, \ldots, K, \mathbf{e}_{K}=$ $(e(i))_{i=0}^{K-1}$, with $\{o b(t), t=1, \ldots, K\}$ being a data set of $K$ observations and $\{x(t), t=1, \ldots, K\}$, the system response starting from an initial condition $x(0)$.

Several studies on parameter estimation and structure identification can be found in the literature. The main problem of these models is to determine techniques to adjust the parameters from experimental data. An analysis of experimental data errors due to parameter sensitivity is given by Boyadjiev and Dimitrova (2005). In the work Verdière et al. (2005) a nonlinear pharmacokinetic model is used and it is analyzed how only one output polynomial provides information on the identifiability of the model; this information is used in numerical parameter estimation. Finally, a good review of identifiability problems and the estimation process is given by Chou and Voit (2013).

This paper is organized as follows. In the next section, we present the stage-structured population model and a background on the mathematical tools used throughout the work. In Section 3, we obtain theoretical results on the identifiability of the parameters involved in the process. Then, we board the estimation problem establishing conditions in order to ensure an estimated value of the parameter from a data set. In Section 4, the analysis of our model leads us to study properties of a sum of rank-one matrices. Then, we establish a recursive process to improve the values of the parameters by addition of more observed data. The results are illustrated with an example. 


\section{Identifiability and estimation of parameters in stage-structured models}

We consider the stage-structured population model including a controlled inflow of elements represented by (1). The transformation matrix

$$
S=\operatorname{diag}\left(1, s_{1}, s_{1} s_{2}, \ldots, s_{1} \cdots s_{n-1}\right)
$$

leads us from the model (1) to a similar system, with $x(t)=S^{-1} \bar{x}(t)$, given by

$$
x(t+1)=A(\mathbf{p}) x(t)+B,
$$

with

$$
A(\mathbf{p})=S^{-1} A S=\left(\begin{array}{ccccc}
a_{1} & a_{2} & \cdots & a_{n-1} & a_{n} \\
1 & \tau_{2} & \cdots & 0 & 0 \\
\vdots & \ddots & \ddots & \vdots & \vdots \\
\vdots & \ddots & \ddots & \tau_{n-1} & \vdots \\
0 & 0 & \cdots & 1 & \tau_{n}
\end{array}\right)
$$

where $a_{1}=\tau_{1}, a_{j}=f_{j} \prod_{i=1}^{j-1} s_{i}, j=2, \ldots, n$. Note that the transformation matrix $S$ preserves the matrix

$$
B=\left(\begin{array}{llll}
b & 0 & \cdots & 0
\end{array}\right)^{T} .
$$

The parameter vector $\mathbf{p}$ is given by $\mathbf{p}=\left(\overline{\mathbf{p}}^{T} \mid \overline{\overline{\mathbf{p}}}^{T}\right)^{T}=$ $\left(a_{1} \cdots a_{n} \mid \tau_{2} \cdots \tau_{n}\right)^{T} \in \mathcal{P}$, where $\mathcal{P}=\overline{\mathcal{P}} \oplus \overline{\mathcal{P}}$, with $\overline{\mathcal{P}}=\left\{(\overline{\mathbf{p}}, O) / \overline{\mathbf{p}}=\left(\bar{p}_{i}\right) \in \mathbb{R}_{+}^{n}, \quad 0 \leq \bar{p}_{1}<1\right\}$ and $\overline{\overline{\mathcal{P}}}$ $=\left\{(O, \overline{\overline{\mathbf{p}}}) / \overline{\overline{\mathbf{p}}}=\left(\overline{\bar{p}}_{i}\right) \in \mathbb{R}_{+}^{n-1} / 0 \leq \overline{\bar{p}}_{i}<1\right\}$.

It is of interest to accurately estimate these parameters. As a first step, we propose to study the identifiability of the system.

It is known that a model is globally identifiable if and only if there exists a single input-output behavior for every parameter set. Therefore, from a fixed structure, we want to test whether the relationship between the parameter sets and the response of the model is unique.

The class of positive structured models $S(\mathbf{p})=$ $(A(\mathbf{p}), B)$ given in (2) has the response

$$
x(t)=A(\mathbf{p})^{t} x(0)+\sum_{i=0}^{t-1} V(i, \mathbf{p}), \quad t \geq 0,
$$

given by the Markov parameters $V(i, \mathbf{p})=A(\mathbf{p})^{i} B, i \geq$ 0 , with matrices $A(\mathbf{p})$ and $B$ given in (3) and (4).

From now on, we consider the initial-value problem

$$
\text { (IVP) }\left\{\begin{array}{l}
x(t+1)=A(\mathbf{p}) x(t)+B, \\
x(0)=x_{1}(0) e_{1} \in \mathbb{R}_{+}^{n}, \text { with } x_{1}(0)>0 .
\end{array}\right.
$$

In the following theorem, we analyze its response.

Theorem 1. For $(\boldsymbol{I V P})$ the response $x(i)=\left(x_{j}(i)\right)_{j=1}^{n}$, for all $1 \leq i<n$, satisfies $x_{j}(i) \geq 0$ if $1 \leq j \leq i$, $x_{i+1}(i)>0$ and $x_{j}(i)=0, i+1<j \leq n$.
Proof. The solution of the system (2) is given by

$$
x(i)=A^{i}(\mathbf{p}) x(0)+\sum_{l=0}^{i-1} A^{l}(\mathbf{p}) B .
$$

From the structure of $A(\mathbf{p})$ and $B=b e_{1}$ given in (3) and (4), choosing $x(0)=x_{1}(0) e_{1}$, we obtain that $x(i)$ is in a polyhedral cone. Specifically, it is a linear combination with nonnegative scalars of the kind

$$
x(i)=\alpha_{1} e_{1}+\cdots+\alpha_{i+1} e_{i+1},
$$

with $\alpha_{j} \geq 0, j=1, \ldots, i$, and $\alpha_{i+1}>0$. Then, $x_{j}(i) \geq$ 0 for $j=1, \ldots i, x_{i+1}(i)>0$ and $x_{j}(i)=0$, for $j=$ $i+1, \ldots, n$.

For linear models there are many well established techniques for analyzing identifiability; see, e.g., the works of Boyadjiev and Dimitrova (2005) or Dion et al. (2003) and the references therein. In our case, to identify the parameters, we consider an initial state $x(0)$ and two parameters $\mathbf{p}, \mathbf{q} \in \mathcal{P}$, and we suppose that $V(j, \mathbf{p})=$ $V(j, \mathbf{q})$. Then, we want to prove that $\mathbf{p}=\mathbf{q}$, that is, the identifiability of the system class $S(\mathbf{p})$. This result is given in the following theorem.

Theorem 2. Consider the stage-structured system class $S(\mathbf{p})=(A(\mathbf{p}), B)$ given by (3) and (4) with $\mathbf{p} \in \mathcal{P} \subseteq \mathbb{R}_{+}^{2 n-1}$. The Markov parameters $V(j, \mathbf{p})=$ $\left(v(j, \mathbf{p})_{\alpha}\right)_{\alpha=1}^{n}, j=1, \ldots, n$, satisfy

$$
\begin{aligned}
v(j, \mathbf{p})_{1} & =\sum_{l=1}^{j} a_{l} v(j-1, \mathbf{p})_{l}, \\
v(j, \mathbf{p})_{\alpha} & =v(j-1, \mathbf{p})_{\alpha-1}+\tau_{\alpha} v(j-1, \mathbf{p})_{\alpha}, \\
\alpha & =2, \ldots, j, \\
v(j, \mathbf{p})_{j+1} & =b, \\
v(j, \mathbf{p})_{\alpha} & =0, \quad \alpha=j+2, \ldots n,
\end{aligned}
$$

and the stage-structured system class $S(\mathbf{p})=(A(\mathbf{p}), B)$ is identifiable.

Proof. Exploiting the form of matrix $A(\mathbf{p})$, we obtain

$$
\begin{aligned}
V(j, \mathbf{p})= & A^{j}(\mathbf{p}) B=A(\mathbf{p}) V(j-1, \mathbf{p}) \\
= & \left(\sum_{l=1}^{n} a_{l} v(j-1, \mathbf{p})_{l},\right. \\
& v(j-1, \mathbf{p})_{1}+\tau_{2} v(j-1, \mathbf{p})_{2}, \\
& \left.\cdots, v(j-1, \mathbf{p})_{n-1}+\tau_{n} v(j-1, \mathbf{p})_{n}\right)^{T} .
\end{aligned}
$$

In addition, a straight recursive calculation allows us to check the expression established in (6). Then, if we consider $V(j, \mathbf{p})=V(j, \mathbf{q})$, we can identify all parameters following the steps of Algorithm 1. 
Algorithm 1. Identification of the parameter $\mathbf{p .}$

Step 1. Input data: $b$, input variable parameters $\mathbf{p}$ and $\mathbf{q}$ such that

$$
\begin{aligned}
& \mathbf{p}=\left(\overline{\mathbf{p}}^{T} \mid \overline{\overline{\mathbf{p}}}^{T}\right)^{T}=\left(a_{1} \cdots a_{n} \mid \tau_{2} \cdots \tau_{n}\right)^{T}, \\
& \mathbf{q}=\left(\overline{\mathbf{q}}^{T} \mid \overline{\overline{\mathbf{q}}}^{T}\right)^{T}=\left(\hat{a}_{1} \cdots \hat{a}_{n} \mid \hat{\tau}_{2} \cdots \hat{\tau}_{n}\right)^{T},
\end{aligned}
$$

where $\overline{\mathbf{p}}, \overline{\mathbf{q}} \in \overline{\mathcal{P}}$ and $\overline{\overline{\mathbf{p}}}, \overline{\overline{\mathbf{q}}} \in \overline{\overline{\mathcal{P}}}$.

Step 2. Construct $A(\mathbf{p}), A(\mathbf{q})$ and $B$ as in (3) and (4).

Step 3. Set $j=1$ :

Step 3.1. Construct the Markov parameters $V(j, \mathbf{p})$ and $V(j, \mathbf{q})$ of the system (2).

Step 3.2. Solve $V(j, \mathbf{p})=V(j, \mathbf{q})$. From (7), it is obtained that $a_{j}=\hat{a}_{j}$ and $\tau_{j}=\hat{\tau}_{j}$ (when $j=1$ only $\left.a_{1}=\hat{a}_{1}\right)$.

Step 3.3. Make $j=j+1$. If $j \leq n$ then return to Step 3.1. Otherwise, go to Step 4.

Step 4. All parameters are identified, $a_{j}=\hat{a}_{j}$ and $\tau_{j}=$ $\hat{\tau}_{j}$, for all $j$. Then $\mathbf{p}=\mathbf{q}$.

Having checked that all the parameters are identifiable, we attack the estimation of their values. Parameter estimation is an important issue in biological and dynamic population systems because it is necessary in order to know the behavior of the dynamic process.

Given the observed experimental data set $\{o b(i) \in$ $\left.\mathbb{R}^{n}, i=1, \ldots, K\right\}$, we consider $e(i)=(o b(i)-x(i))$ as the error between the observed value and the obtained value by the simulation of the given model at time $i, i=$ $1, \ldots, K$. Rewriting the system (2),

$$
x(t+1)=M(t) \mathbf{p}+N(t), \quad t \geq 0 .
$$

From $d(i)=o b(i)-N(i-1), i=1, \ldots, K$ and $M(i), i=0, \ldots, K-1$, we construct the following stacked matrices:

$$
d_{K}=\operatorname{col}(d(i))_{i=1}^{K}, \quad H_{K}=\operatorname{col}(M(i))_{i=0}^{K-1} .
$$

We look for the value of the parameter which minimizes the quadratic error

$$
\begin{aligned}
J_{K}(\mathbf{p}) & =\frac{1}{2} \sum_{i=1}^{K} e(i)^{T} e(i) \\
& =\frac{1}{2} \mathbf{e}_{K}^{T} \mathbf{e}_{K}=\frac{1}{2}\left(d_{K}-H_{K} \mathbf{p}\right)^{T}\left(d_{K}-H_{K} \mathbf{p}\right) .
\end{aligned}
$$

This minimizer $\mathbf{p}$ satisfies

$$
\frac{\partial J_{K}(\mathbf{p})}{\partial \mathbf{p}}=H_{K}^{T} H_{K} \mathbf{p}-H_{K}^{T} d_{K}=0 .
$$

Note that the vector $\mathbf{p}$ that minimizes the function $J_{K}(\mathbf{p})$ is given as follows. If $H_{K}^{T} H_{K}$ is nonsingular, then $\mathbf{p}=$
$\left(H_{K}^{T} H_{K}\right)^{-1} H_{K}^{T} d_{K}$, and if it is singular, then $\mathbf{p}=H_{K}^{\dagger} d_{K}$, where $\dagger$ denotes the Moore-Penrose generalized inverse matrix.

From the system (2) and the structure of matrix $A(\mathbf{p})$ given in (3), we obtain

$$
\begin{aligned}
& M(t)=\left(\begin{array}{cc}
x^{T}(t) & O \\
O & D_{t}
\end{array}\right), \\
& N(t)=\left(b x_{1}(t) x_{2}(t) \cdots x_{n-1}(t)\right)^{T},
\end{aligned}
$$

with

$$
\begin{aligned}
x(t) & =\left(x_{i}(t)\right)_{i=1}^{n}, \\
D_{t} & =\operatorname{diag}\left(x_{2}(t), \ldots, x_{n}(t)\right) .
\end{aligned}
$$

Then,

$$
H_{K}^{T} H_{K}=\left(\begin{array}{cc}
X_{k} X_{K}^{T} & O \\
O & Y_{K}^{T} Y_{K}
\end{array}\right), \quad d_{K}=\left(\begin{array}{c}
\bar{d}_{K} \\
\bar{d}_{K}
\end{array}\right),
$$

with

$$
\begin{aligned}
X_{K} & =(x(0) \cdots x(K-1)), \\
Y_{K} & =\operatorname{col}\left(D_{i}\right)_{i=0}^{K-1}, \\
\bar{d}_{K} & =\operatorname{col}\left(d_{1}(i)\right)_{i=1}^{K}, \\
\overline{\bar{d}}_{K} & =\operatorname{col}\left(\left(\overline{\bar{d}}_{K}\right)_{i}\right)_{i=1}^{K},
\end{aligned}
$$

with $\left(\overline{\bar{d}}_{K}\right)_{i}=\operatorname{col}\left(d_{j}(i)\right)_{j=2}^{n}$.

Taking into account the structure of above matrices and $\mathbf{p}=\left(\overline{\mathbf{p}}^{T} \mid \overline{\mathbf{p}}^{T}\right)^{T}$, we have the matrix equation $H_{K}^{T} H_{K} \mathbf{p}=H_{K}^{T} d_{K}$, which is uncoupled into two matrix equations:

$$
\begin{array}{lll}
S_{K} \overline{\mathbf{p}}=X_{K} \bar{d}_{K} & \text { with } \quad S_{K}=X_{K} X_{K}^{T}, \\
T_{K} \overline{\overline{\mathbf{p}}}=Y_{K}^{T} \overline{\bar{d}}_{K} & \text { with } \quad T_{K}=Y_{K}^{T} Y_{K} .
\end{array}
$$

The analysis of the solutions of these equations leads to the following results. First, we consider the estimation problem only referring to the structure of the coefficient matrices $A(\mathbf{p})$ and $B$ :

\section{(PEP1) Parameter estimation problem without re- strictions on $\mathbf{p :}$}

Find $\boldsymbol{p}=\left(\overline{\boldsymbol{p}}^{T} \mid \overline{\overline{\boldsymbol{p}}}^{T}\right)^{T}$ satisfying the system of equations (10) and (11).

(PEP1) has a solution for any number of observations $K$. Construct the matrix $X_{K}=(x(0) \cdots x(K-1))$. Using the form of $x(i)$ given in (5), matrix $S_{K}$ is singular if $K<n$ and nonsingular if $K \geq n$. When $K<n$, we are using the Moore-Penrose inverse of $S_{K}$ to solve the estimation problem $S_{K} \overline{\mathbf{p}}=X_{K} \bar{d}_{K}$, and $S_{n}^{-1}$ when $K=n$. We give only the result for the case when the number of observations $K$ exceeds $n$. 
Theorem 3. Consider (IVP) and an observed data set $\{o b(i)\}_{i=1}^{K}$ with $K \geq n$. Then (PEP1) has a unique solution given by

$$
\begin{gathered}
\overline{\boldsymbol{p}}=S_{K}^{-1} X_{K} \bar{d}_{K}, \\
\overline{\overline{\boldsymbol{p}}}=T_{K}^{-1} Y_{K}^{T} \overline{\bar{d}}_{K} .
\end{gathered}
$$

Proof. Construct the matrix

$$
X_{K}=(x(0) \cdots x(K-1)) .
$$

Using the form of $x(i)$ given in (5) we can ensure that $X_{K}$ is of full row rank. Hence, matrix $S_{K}=X_{K} X_{K}^{T}$ is nonsingular. Then $\overline{\mathbf{p}}=S_{K}^{-1} X_{K} \bar{d}_{K}$.

From Theorem 1 for all time indices $i, 1 \leq i \leq n$, we have that, for $j=2, \ldots, n$, the entry $x_{j}(i+1)$ is positive. Then, we can assure that $Y_{K}$ is of full column rank. Thus, $T_{K}=Y_{K}^{T} Y_{K}$ is nonsingular and the assertion follows. That is, $\overline{\overline{\mathbf{p}}}=T_{K}^{-1} Y_{K}^{T} \overline{\bar{d}}_{K}$.

Note that the parameter estimation problem associated with our model is more restrictive. In particular, we need a parameter vector $\mathbf{p}=\left(\overline{\mathbf{p}}^{T} \mid \overline{\overline{\mathbf{p}}}^{T}\right)^{T}$ which satisfies the necessary conditions to represent the evolution of a structured population by stages. Thus, the problem will be formulated as follows:

\section{(PEP2) Parameter estimation problem with $\mathbf{p} \in \mathcal{P}$ :}

Find $\boldsymbol{p}=\left(\overline{\boldsymbol{p}}^{T} \mid \overline{\overline{\boldsymbol{p}}}^{T}\right)^{T}$ satisfying the system of equations (10) and (11), and fulfilling $\boldsymbol{p}=\left(\overline{\boldsymbol{p}}^{T} \mid \overline{\overline{\boldsymbol{p}}}^{T}\right)^{T} \in$ $\mathcal{P}$, with $\mathcal{P}=\overline{\mathcal{P}} \oplus \overline{\overline{\mathcal{P}}}$,

$$
\begin{aligned}
& \overline{\mathcal{P}}=\left\{(\overline{\mathbf{p}}, O) / \overline{\mathbf{p}}=\left(\bar{p}_{i}\right) \in \mathbb{R}_{+}^{n}, \quad 0 \leq \bar{p}_{1}<1\right\}, \\
& \overline{\overline{\mathcal{P}}}=\left\{(O, \overline{\overline{\mathbf{p}}}) / \overline{\overline{\mathbf{p}}}=\left(\overline{\bar{p}}_{i}\right) \in \mathbb{R}_{+}^{n-1}, \quad 0 \leq \overline{\bar{p}}_{i}<1\right\} .
\end{aligned}
$$

Lemma 1. Consider (IVP) and an observed data set $\{o b(i)\}_{i=1}^{K}$ with $1 \leq K \leq n$. Then the equation $S_{K} \overline{\boldsymbol{p}}=$ $X_{K} \bar{d}_{K}$ has a solution $\overline{\boldsymbol{p}}=\left(\bar{p}_{i}\right)_{i=1}^{n}$, satisfying $\bar{p}_{i} \geq 0$ and $0 \leq \bar{p}_{1}<1$ if and only if the following conditions are satisfied:

(i) $\sum_{j=1}^{i}(-1)^{i-j} M_{i-j}(j-1) x_{1}(0)^{j-1} d_{1}(j) \geq 0, i=$ $1, \ldots, K$, with $M_{\alpha}(\beta)$ given in (13) and $M_{0}(\beta)=1$.

(ii) $0 \leq d_{1}(1)<x_{1}(0)$.

Proof. For the observed data set in question and from the initial state $x(0)=x_{1}(0) e_{1} \in \mathbb{R}_{+}^{n}$ with $x_{1}(0)>0$, the matrix $X_{K}$ given in (9) is

$$
X_{K}=\left(\begin{array}{c}
U_{K} \\
O
\end{array}\right)
$$

with

$$
U_{K}=\left(\begin{array}{cccc}
x_{1}(0) & x_{1}(1) & \cdots & x_{1}(K-1) \\
0 & x_{2}(1) & \cdots & x_{2}(K-1) \\
\vdots & \vdots & \cdots & \vdots \\
0 & 0 & \cdots & x_{K}(K-1)
\end{array}\right),
$$

nonsingular. When $K<n$, we are using the Moore-Penrose inverse of $S_{K}$ to solve the estimation problem $S_{K} \overline{\mathbf{p}}=X_{K} \bar{d}_{K}$, and $S_{n}^{-1}$ when $K=n$,

$$
\begin{aligned}
& S_{K}^{\dagger} X_{K}=\left(X_{K}^{T}\right)^{\dagger}=\left(\begin{array}{c}
\left(U_{K}^{T}\right)^{-1} \\
O
\end{array}\right), \\
& S_{n}^{-1} X_{n}=U_{n}^{-1} .
\end{aligned}
$$

From Theorem $1, K \leq n$, and from the expressions given in (14), we consider the inverse matrix of $\left(U_{K}^{T}\right)^{-1}$, which is a lower triangular matrix. Then, to calculate its inverse matrix, we can use the explicit formula given by Baliarsingh and Dutta (2015), obtaining its $(i, j)$-th entry given by

$$
\begin{aligned}
& \left(\left(U_{K}^{T}\right)^{-1}\right)_{i j} \\
& \quad= \begin{cases}\frac{(-1)^{i-j} M_{i-j}(j-1)}{\left(x_{1}(0)\right)^{i-j+1}}, & 1 \leq j<i, \\
\frac{1}{x_{1}(0)}, & j=i, \\
0, & j>i,\end{cases}
\end{aligned}
$$

for $i, j=1, \ldots, K$, with $M_{\alpha}(\beta)$ given in (13).

Denoting by $\bar{p}_{i}$ the $i$-th entry of $\overline{\mathbf{p}}$ and by $\left(\left(U_{K}^{T}\right)^{-1}\right)_{i}$ the $i$-th row $\left(U_{K}^{T}\right)^{-1}$, we observe that

$$
\begin{aligned}
\bar{p}_{i}= & \left(\left(U_{K}^{T}\right)^{-1}\right)_{i} \bar{d}_{K} \\
= & \sum_{j=1}^{i-1}(-1)^{i-j} \frac{M_{i-j}(j-1)}{\left(x_{1}(0)\right)^{i-j+1}} d_{1}(j) \\
& +\frac{1}{x_{1}(0)} d_{1}(i), \quad i=1, \ldots, K,
\end{aligned}
$$

$$
\bar{p}_{i}=0, \quad i=K+1, \ldots, n .
$$

Then, the condition (i) is satisfied if and only if $\left(\left(U_{K}^{T}\right)^{-1}\right)_{i} \bar{d}_{K} \geq 0$, that is, $\bar{p}_{i} \geq 0$, for $i=1, \ldots, n$. Moreover, we have that

$$
\left(\left(U_{K}^{T}\right)^{-1}\right)_{1}=\left(\frac{1}{x_{1}(0)} 0 \cdots 0\right) .
$$

Then $\bar{p}_{1}=d_{1}(1) / x_{1}(0)$ and $0 \leq \bar{p}_{1}<1$ if and only if the condition given in (ii) holds.

Lemma 2. Consider (IVP) and an observed data set $\{o b(i)\}_{i=1}^{K}$ with $K \geq 1$. Setting $x_{K}^{(i)}=\operatorname{col}\left(x_{i}(j)\right)_{j=0}^{K-1}$ and $d_{K}^{(i)}=\operatorname{col}\left(d_{i}(j)\right)_{j=1}^{K} i=2, \ldots, n$, we have that $T_{K} \overline{\overline{\boldsymbol{p}}}=Y_{K}^{T} \overline{\bar{d}}_{K}$ has a solution $\overline{\overline{\boldsymbol{p}}}$ satisfying $0 \leq \overline{\bar{p}}_{i}<1$, with $\overline{\overline{\boldsymbol{p}}}=\left(\overline{\bar{p}}_{i}\right)_{i=1}^{n-1}$ if and only if

(iii) $0 \leq\left(x_{K}^{(i)}\right)^{T} d_{K}^{(i)}<\left\|x_{K}^{(i)}\right\|_{2}^{2}$

Proof. From the definition of matrix $Y_{K}$ in (9), we have

$$
Y_{K}^{\dagger}=\operatorname{diag}\left(\left\|x_{K}^{(2)}\right\|_{2}^{-2}, \ldots,\left\|x_{K}^{(K)}\right\|_{2}^{-2}\right) Y_{K}^{T} .
$$




$$
M_{\alpha}(\beta)=\left|\begin{array}{ccccc}
x_{\beta+1}(\beta+1) & x_{\beta+2}(\beta+1) & 0 & \cdots & 0 \\
x_{\beta+1}(\beta+2) & x_{\beta+2}(\beta+2) & x_{\beta+3}(\beta+2) & \cdots & 0 \\
\vdots & \vdots & \vdots & \ddots & \vdots \\
x_{\beta+1}(\beta+\alpha) & x_{\beta+2}(\beta+\alpha) & x_{\beta+3}(\beta+\alpha) & \cdots & x_{\beta+\alpha}(\beta+\alpha)
\end{array}\right|
$$

Then, from Eqn. (11), we have that $\overline{\bar{p}}_{i}, i=1, \ldots, n-$ 1 , is given by

$$
\begin{array}{ll}
\overline{\bar{p}}_{i}=\left\|x_{K}^{(i+1)}\right\|_{2}^{-2} \sum_{j=0}^{K-1} x_{i+1}(j) d_{i+1}(j+1) \\
\quad \text { if } i=1, \ldots, K-1, \\
\overline{\bar{p}}_{i}=0 & \text { if } i=K, \ldots, n-1,
\end{array}
$$

when $K<n$. If $K \geq n$, the expression (16) holds for $i=1, \ldots, n-1$. Then, $0 \leq \overline{\bar{p}}_{i}<1$ if and only if

$$
\begin{aligned}
0 & \leq\left(x_{i+1}(0) \cdots x_{i+1}(K-1)\right)^{T}\left(\begin{array}{c}
d_{i+1}(1) \\
\vdots \\
d_{i+1}(K)
\end{array}\right) \\
& <\left(x_{i+1}(0) \cdots x_{i+1}(K-1)\right)^{T}\left(\begin{array}{c}
x_{i+1}(0) \\
\vdots \\
x_{i+1}(K-1)
\end{array}\right) .
\end{aligned}
$$

\section{Writing}

$$
x_{K}^{(i)}=\operatorname{col}\left(x_{i}(j)\right)_{j=0}^{K-1}, \quad d_{K}^{(i)}=\operatorname{col}\left(d_{i}(j)\right)_{j=1}^{K},
$$

$i=2, \ldots, n$, we have the condition given in (iii).

Note that, under the conditions (i)-(iii), the parameter $\mathbf{p}=\left(\overline{\mathbf{p}}^{T} \mid \overline{\overline{\mathbf{p}}}^{T}\right)^{T}$ belongs to $\mathcal{P}$, with $\mathcal{P}=\overline{\mathcal{P}} \oplus \overline{\overline{\mathcal{P}}}$, $\overline{\mathcal{P}}=\left\{\left(\bar{p}_{i}\right) \in \mathbb{R}_{+}^{n} / 0 \leq \bar{p}_{1}<1\right\}$ and $\overline{\overline{\mathcal{P}}}=\left\{\left(\overline{\bar{p}}_{i}\right) \in\right.$ $\left.\mathbb{R}_{+}^{n-1} / 0 \leq \overline{\bar{p}}_{i}<1\right\}$. As a direct consequence of the previous lemmas, we give the following result.

Theorem 4. Consider (IVP) and an observed data set $\{o b(i)\}_{i=1}^{K}$ with $1 \leq K \leq n$. The parameter vector $\boldsymbol{p}=$ $\left(\overline{\boldsymbol{p}}^{T} \mid \overline{\overline{\boldsymbol{p}}}^{T}\right)^{T}$ given in (15) and (16) solves (PEP2) for $K \leq$ $n$ if and only if the conditions ( $i$ )-(iii) given in Lemmas 1 and 2 hold.

Remark 1. When $k>n$, the matrix $X_{K}$ is a full-rank matrix, and we move to the next section the study of that case.

\section{Recursive process using inversion of a sum of rank-one matrices}

The matrix $S_{i}=X_{i} X_{i}^{T}$ with $X_{i}=(x(0) \cdots x(i-1))$ is a sum of rank-one matrices,

$$
\begin{aligned}
S_{1} & =x(0) x^{T}(0), \\
S_{i+1} & =X_{i+1} X_{i+1}^{T} \\
& =\sum_{l=0}^{i} x(l) x^{T}(l)=S_{i}+x(i) x^{T}(i), \quad i \geq 1 .
\end{aligned}
$$

This fact motives the study of the inverse of a sum of rank-one matrices. It is known (Ljung and Soderstrom, 1983) that, given $S \in \mathbb{R}^{n \times n}$ and $v \in \mathbb{R}^{n}$, if $S$ is nonsingular, then $S+v v^{T}$ is also nonsingular and its inverse matrix is given by

$$
\left(S+v v^{T}\right)^{-1}=S^{-1}-\frac{S^{-1} v v^{T} S^{-1}}{1+v^{T} S^{-1} v} .
$$

We are now able to establish a recursive process in order to improve the estimated value of the parameter vector $\mathbf{p}$ by successive construction of an inverse of a matrix, which is formed as a sum of rank-one matrices.

We assume that $K \geq n$, and we study (PEP2) when a new observed data piece $o b(K+1)$ is obtained.

Given a $K$ observed data set, $\{o b(i), i=1, \ldots, K\}$, we have an estimated value, $\mathbf{p}_{K}$, of the parameter vector and we construct the system (2) with matrices $A\left(\mathbf{p}_{K}\right)$ and $B$ given in (3) and (4), and define the following matrices:

$$
\mathcal{A}_{K}=\frac{S_{K}^{-1} x(K)}{1+\alpha_{K}}, \quad \mathcal{B}_{K}=\left(I+D_{K}^{2} T_{K}^{-1}\right)^{-1} D_{K},
$$

with $\alpha_{K}=x^{T}(K) S_{K}^{-1} x(K)$. To improve the approximated value of parameter $\mathbf{p}$, we add one observation $o b(K+1)$ and fit the mathematical model to the $(K+1)$-element observed data set.

Theorem 5. Let $K(K \geq n)$ be an observed data set $\{o b(i)\}_{i=1}^{K}$. Consider the assumptions of Theorem 3 and $\boldsymbol{p}_{K}=\left(\overline{\boldsymbol{p}}_{K}^{T} \mid \overline{\overline{\boldsymbol{p}}}_{K}^{T}\right)^{T}$ as the parameter solving (PEP1) for $K$. Given a new observed data piece ob $(K+1)$, the parameter vector $\boldsymbol{p}_{K+1}=\left(\overline{\boldsymbol{p}}_{K+1}^{T} \mid \overline{\overline{\boldsymbol{p}}}_{K+1}^{T}\right)^{T}$ given by

$$
\begin{gathered}
\overline{\boldsymbol{p}}_{K+1}=\left(I-\mathcal{A}_{K} x^{T}(K)\right) \overline{\boldsymbol{p}}_{K}+\mathcal{A}_{K} d_{1}(K+1), \\
\overline{\overline{\boldsymbol{p}}}_{K+1}=\mathcal{B}_{K} D_{K}^{-1}\left(\overline{\overline{\boldsymbol{p}}}_{K}+T_{K}^{-1} D_{K}\left(\begin{array}{c}
d_{2}(K+1) \\
\vdots \\
d_{n}(K+1)
\end{array}\right)\right)
\end{gathered}
$$

solves (PEP1) for $K+1$.

Proof. Given the observed data $\{o b(i)\}_{i=1}^{K+1}$, we have that $\mathbf{p}_{K+1}=\left(\overline{\mathbf{p}}_{K+1}^{T} \mid \overline{\mathbf{p}}_{K+1}^{T}\right)^{T}$ solves (PEP1) for $K+1$ if it is the solution of the system

$$
\begin{aligned}
& S_{K+1} \overline{\mathbf{p}}=X_{K+1} \bar{d}_{K+1}, \\
& T_{K+1} \overline{\overline{\mathbf{p}}}=Y_{K+1}^{T} \overline{\bar{d}}_{K+1} .
\end{aligned}
$$


By construction of matrices $S_{i}, X_{i}$ and $\bar{d}_{i}$, we have

$$
\begin{aligned}
\overline{\mathbf{p}}_{K+1}= & S_{K+1}^{-1} X_{K+1} \bar{d}_{K+1} \\
= & \left(S_{K}^{-1}-\frac{S_{K}^{-1} x(K) x^{T}(K) S_{K}^{-1}}{1+\alpha_{K}}\right) \\
& \times\left(X_{K} x(K)\right)\left(\begin{array}{c}
\bar{d}_{K} \\
d_{1}(K+1)
\end{array}\right) \\
= & \left(I-\frac{S_{K}^{-1} x(K) x^{T}(K)}{1+\alpha_{K}}\right) \underbrace{S_{K}^{-1} X_{K} \bar{d}_{K}}_{\overline{\mathbf{p}}_{K}} \\
& +S_{K}^{-1} x(K) d_{1}(K+1) \\
& -\frac{S_{K}^{-1} x(K) \overbrace{}^{T}(K) S_{K}^{-1} x(K)}{1+\alpha_{K}} d_{1}(K+1) \\
= & \left(I-\frac{S_{K}^{-1} x(K) x^{T}(K)}{1+\alpha_{K}}\right) \overline{\mathbf{p}}_{K} \\
& +\frac{S_{K}^{-1} x(K)}{1+\alpha_{K}} d_{1}(K+1) .
\end{aligned}
$$

Further, using the definitions of $T_{i}, Y_{i}, D_{i}$ and $\overline{\bar{d}}_{i}$, as

$$
\begin{aligned}
T_{K+1}^{-1} & =\left(\left(Y_{K}^{T} D_{K}\right)\left(\begin{array}{c}
Y_{K} \\
D_{K}
\end{array}\right)\right)^{-1} \\
& =\left(T_{K}+D_{K}^{2}\right)^{-1}=\left(I+T_{K}^{-1} D_{K}^{2}\right)^{-1} T_{K}^{-1},
\end{aligned}
$$

we obtain

$$
\begin{aligned}
& \overline{\overline{\mathbf{p}}}_{K+1} \\
& =T_{K+1}^{-1} Y_{K+1}^{T} \overline{\bar{d}}_{K+1} \\
& =\left(I+T_{K}^{-1} D_{K}^{2}\right)^{-1} T_{K}^{-1}\left(Y_{K}^{T} D_{K}\right)\left(\begin{array}{c}
\overline{\bar{d}}_{K} \\
d_{2}(K+1) \\
\vdots \\
d_{n}(K+1)
\end{array}\right) \\
& =\left(I+T_{K}^{-1} D_{K}^{2}\right)^{-1} \\
& \times(\underbrace{T_{K}^{-1} Y_{K}^{T} \overline{\bar{d}}_{K}}_{\overline{\mathbf{p}}_{K}}+T_{K}^{-1} D_{K}\left(\begin{array}{c}
d_{2}(K+1) \\
\vdots \\
d_{n}(K+1)
\end{array}\right))
\end{aligned}
$$

Hence, using the matrices $\mathcal{A}_{K}$ and $\mathcal{B}_{K}$ defined in (17), the parameter vector $\mathbf{p}_{K+1}=\left(\overline{\mathbf{p}}_{K+1}^{T} \mid \overline{\mathbf{p}}_{K+1}^{T}\right)^{T}$ given in (18) solves (PEP1) for $K+1$.

Analyzing the expressions given in (18), if the parameter $\overline{\mathbf{p}}_{K} \in \mathcal{P}$, we can prove that the conditions

(iv) $\overline{\mathbf{p}}_{K}+\mathcal{A}_{K} \beta_{K} \geq O$,

(v) $-\bar{p}_{K, 1} \leq\left(\mathcal{A}_{K}\right)_{1} \beta_{K}<1-\bar{p}_{K, 1}$,

guarantee that $\overline{\mathbf{p}}_{K+1}$ is nonnegative with the first entry less than 1, where $\beta_{K}=\left(d_{1}(K+1)-x^{T}(K) \overline{\mathbf{p}}_{K}\right)$. Also, the condition (vi) $\frac{\left(d_{i+1}(K+1)-x_{i+1}(K)\right) x_{i+1}(K)}{\left\|x_{K}^{(i+1)}\right\|_{2}^{2}}<\left(1-\overline{\bar{p}}_{K, i}\right)$, for all $i=1, \ldots, n-1$,

is equivalent to $0 \leq \overline{\bar{p}}_{K+1, i}<1, i=1, \ldots, n-1$.

Thus, under these conditions, the parameter vector given in (18) also solves (PEP2) for $K+1$. This fact is expressed in the following theorem.

Theorem 6. Let $K(K \geq n)$ be an observed data set $\{o b(i)\}_{i=1}^{K}$. Under the assumptions of Theorem 3 let $\boldsymbol{p}_{K}=\left(\overline{\boldsymbol{p}}_{K}^{T} \mid \overline{\overline{\boldsymbol{p}}}_{K}^{T}\right)^{T}$ be a parameter solving (PEP2) for $K$. Given a new observed data piece ob $(K+1), \boldsymbol{p}_{K+1}$ given in (18) solves (PEP2) for $K+1$ if and only if the conditions (iv)-(vi) are satisfied.

Last, using the 2-norm, we show some conditions to ensure the closeness between the two approaches. From (18) we have

$$
\begin{aligned}
& \left\|\overline{\mathbf{p}}_{K+1}-\overline{\mathbf{p}}_{K}\right\|_{2} \\
& =\left\|\left(I-\mathcal{A}_{K} x^{T}(K)\right) \overline{\mathbf{p}}_{K}+\mathcal{A}_{K} d_{1}(K+1)-\overline{\mathbf{p}}_{K}\right\|_{2} \\
& =\left\|\mathcal{A}_{K}\left(-x^{T}(K) \overline{\mathbf{p}}_{K}+d_{1}(K+1)\right)\right\|_{2} \\
& \leq\left\|\mathcal{A}_{K}\right\|_{2}\left|-x^{T}(K) \overline{\mathbf{p}}_{K}+d_{1}(K+1)\right| \\
& =\left\|\mathcal{A}_{K}\right\|_{2}\left|o b_{1}(K+1)-x_{1}(K+1)\right|
\end{aligned}
$$

and

$$
\begin{aligned}
\left\|\overline{\overline{\mathbf{p}}}_{K+1}-\overline{\overline{\mathbf{p}}}_{K}\right\|_{2} \\
=\|\left(\left(I+T_{K}^{-1} D_{K}^{2}\right)^{-1}-I\right) \overline{\overline{\mathbf{p}}}_{K} \\
\quad+\left(I+T_{K}^{-1} D_{K}^{2}\right)^{-1} T_{K}^{-1} D_{K} \overline{\bar{d}}(k+1) \|_{2} \\
=\left\|\mathcal{B}_{K} T_{K}^{-1}\left(-D_{K} \overline{\overline{\mathbf{p}}}_{K}+\overline{\bar{d}}(k+1)\right)\right\|_{2} \\
\leq\left\|\mathcal{B}_{K} T_{K}^{-1}\right\|_{2}\left\|-D_{K} \overline{\overline{\mathbf{p}}}_{K}+\overline{\bar{d}}(k+1)\right\|_{2} \\
=\left\|\mathcal{B}_{K} T_{K}^{-1}\right\|_{2}\|\overline{\bar{x}}(K+1)-\overline{\bar{o}}(K+1)\|_{2},
\end{aligned}
$$

with $\left.\overline{\bar{\sigma}}(K+1)=\operatorname{col}\left(\sigma_{i}(K+1)\right)\right)_{i=2}^{n}$, and $\sigma=x, d$, ob. Thus, given $\epsilon>0$ and a new observed data piece $o b(K+$ 1) such that

$$
\begin{aligned}
& \left|x_{1}(K+1)-o b_{1}(K+1)\right|<\frac{\epsilon}{2\left\|\mathcal{A}_{K}\right\|_{2}}, \\
& \|\overline{\bar{x}}(K+1)-\overline{\overline{o b}}(K+1)\|_{2}<\frac{\epsilon}{2\left\|\mathcal{B}_{K} T_{K}^{-1}\right\|_{2}},
\end{aligned}
$$

we have

$$
\left\|\mathbf{p}_{K+1}-\mathbf{p}_{K}\right\| \leq\left\|\overline{\mathbf{p}}_{K+1}-\overline{\mathbf{p}}_{K}\right\|+\left\|\overline{\overline{\mathbf{p}}}_{K+1}-\overline{\overline{\mathbf{p}}}_{K}\right\|_{2}<\epsilon .
$$

From now on we shall write

$$
m_{K}=\max \left\{\left\|\mathcal{A}_{K}\right\|_{2},\left\|\mathcal{B}_{K} T_{K}^{-1}\right\|_{2}\right\}
$$

with $\mathcal{A}_{K}$ and $\mathcal{B}_{K}$ given in (17). 
Theorem 7. Let $K \geq n$ and the assumptions of Theorem 3 hold. Consider observed data $\{o b(i)\}_{i=1}^{K}$ and $\boldsymbol{p}_{K}$ solving (PEP2) for $K$. Given $\epsilon>0$ and a new observed data piece ob $(K+1)$ such that

$$
\|x(K+1)-o b(K+1)\|_{2}<\frac{\epsilon}{m_{k}},
$$

with $m_{K}$ given in (24) we have, $\left\|\boldsymbol{p}_{K+1}-\boldsymbol{p}_{K}\right\|_{2}<\epsilon, \boldsymbol{p}_{K+1}$ being the estimated value given in (18).

Proof. Joining expressions given in (18) and rewriting as in (21) and (22), we obtain

$$
\begin{aligned}
& \left\|\mathbf{p}_{K+1}-\mathbf{p}_{K}\right\|_{2} \\
& =\left\|\left(\begin{array}{cc}
\mathcal{A}_{K} & O \\
O & \mathcal{B}_{K} T_{K}^{-1}
\end{array}\right)(o b(K+1)-x(K+1))\right\|_{2} \\
& \leq m_{K}\|x(K+1)-o b(K+1)\|_{2}<\epsilon .
\end{aligned}
$$

The recursive process to improve the estimated value of the parameter vector, fulling also the restrictions of (PEP2) is shown as Algorithm 2.

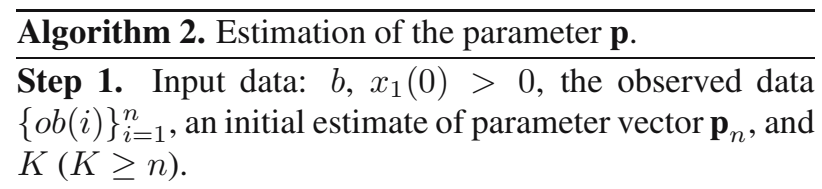

Step 2. Construct the vector $x(0)=\left(\begin{array}{llll}x_{1}(0) & 0 & \cdots & 0\end{array}\right)^{T}$, $A\left(\mathbf{p}_{n}\right)$ and $B$ as in (3) and (4).

For $i=1$ to $n$, we construct $x(i)=A\left(\mathbf{p}_{n}\right) x(i-1)+B$.

Step 3. Calculate $X_{n}, Y_{n}, \bar{d}_{n}, \overline{\bar{d}}_{n}, S_{n}$ and $T_{n}$ as in (9).

Step 4. Set $j=1$.

Step 5. Input observed data $o b(n+j)$.

Step 5.1. Calculate $\overline{\mathbf{p}}_{n+j}$ and $\overline{\overline{\mathbf{p}}}_{n+j}$ as in (18).

Step 5.2. Check the conditions (iv)-(vi). If some of them do not hold, then go to Step 6. Otherwise, go to Step 5.3.

Step 5.3. For $i=1, \ldots, n+j$, construct $x(i)=$ $A\left(\mathbf{p}_{n+j}\right) x(i-1)+B$ and $X_{n+j}, Y_{n+j}, \bar{d}_{n+j}, \overline{\bar{d}}_{n+j}$, $S_{n+j}$ and $T_{n+j}$.

Step 5.4. Set $j=j+1$. If $n+j \leq K$, return to Step 5.1. Otherwise, go to Step 6.

Step 6. The process is finished.

3.1. Illustrative example. The aim of this part is to discuss estimation of the parameters for an element of $S(\mathbf{p})$ in the particular case of a population with three stages. In this model, the individuals that do not reach the desired maturity in a step of time will remain in the same class and will not advance to the next one.

We start from the fact that the initial observed data are known and we have simulated an observed data set to analyze the reliability of the above algorithm.

In our population model, $f_{j}$ : the fertility parameters, $s_{j}$ : the survival rates growing into the next stage and $\tau_{j}$ the survival rates remaining in the same stage, are given by $f_{1}=0, f_{2}=0, f_{3}=3, s_{1}=0.6, s_{2}=$ $0.7, \tau_{1}=0.17, \tau_{2}=0.25, \tau_{3}=0.86$. Taking into account the discussion of the previous sections and making the relevant transformations, we have that $\mathbf{p}=$ $(0.17,0,1.26,0.25,0.86)$. We consider an initial condition which only has individuals of the first class, in particular, given by $x^{T}(0)=(4000,0,0)$, and we suppose the process is altered, in each step, by an entry of $b=400$ individuals of new population at the first stage. Then we simulate the process established in the above algorithm. Starting from the fact that the estimated value of the parameter from three observations obtained is

$\mathbf{p}_{3}=(0.1625,0.0105745,1.24467,0.247957,0.8625)$,

new observations are added. The obtained results are displayed in Fig. 11 These represent the evolution of the parameter estimates for the first $K=15$ observations. The difficulty of finding approximations to specific data is well known, especially when there are some altered data. Nevertheless, our observed data set satisfies the conditions of the theorems given in the last section. Therefore, using the 2-norm, it is observed how close two consecutive estimates of the parameter are.

\section{Conclusions}

A class $S(p)$ of stage-structured population models with unknown parameters was considered. We proposed an iterative method to analyze if the parameters can be uniquely recovered from the observed data. Using this method, we ensured the identifiability of the models in $S(p)$. This fact guarantees that the model parameters can be estimated under ideal conditions. We solved the estimation problem with or without restrictions on the parameters by minimizing the least squares criterion. The novelty that our proposal presents is the use of the structure of the matrices that describe the models of the $S(p)$ class. Thus, these matrices were analyzed and results were obtained that allow us to improve the estimation procedure. In the analysis of the estimation of parameters, we gave some conditions to ensure the restrictions on the parameters, both for a number of observations smaller and larger than the size of the state vector. Finally, we solved the problem of adjusting the parameters from experimental data given a boundary that ensures that two consecutive approximations are sufficiently close. 

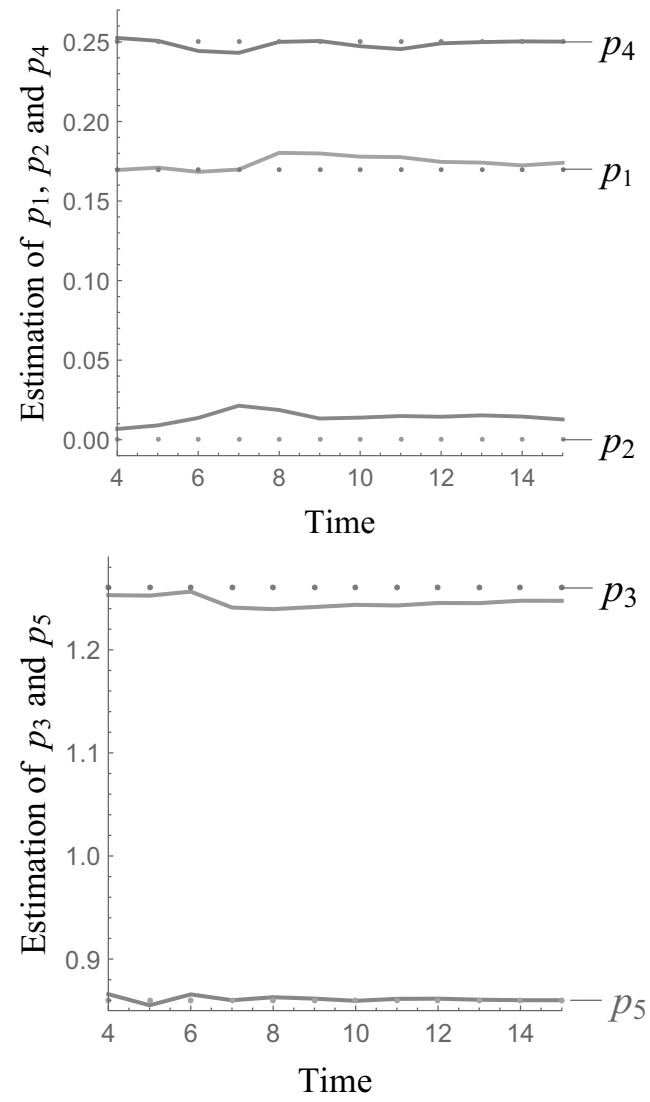

Fig. 1. Estimated values of the parameter $\mathbf{p}=\left(p_{i}\right)_{i=1}^{5}$ from the iterative process when $K=15$.

\section{Acknowledgment}

The authors appreciate the comments and suggestions from the reviewers, which have helped to improve the paper.

This work has been partially supported by the Spanish grant MTM2013-43678-P.

\section{References}

Baliarsingh, P. and Dutta, S. (2015). On an explicit formula for inverse of triangular matrices, Journal of the Egyptian Mathematical Society 23(1): 297-302.

Berman, A. and Plemmons, R. (1994). Nonnegative Matrices in the Mathematical Sciences, SIAM, Philadelphia, PA.

Boyadjiev, C. and Dimitrova, E. (2005). An iterative method for model parameter identification, Computers and Chemical Engineering 29(1): 941-948.

Cantó, B., Coll, C. and Sánchez, E. (2014). On stability and reachability of perturbed positive systems, Advances in Difference Equations 296(1): 1-11.

Cao, H. and Zhou, Y. (2012). The discrete age-structured SEIT model with application to tuberculosis transmission in China, Mathematical and Computer Modelling 55(3-4): 385-395.
Carnia, E., Sylviani, S., Wirmas, M. and Supriatna, A. (2015) Modeling the killer whale Orcinus orca via the Lefkovitch matrix, 3rd International Conference on Chemical, Agricultural and Medical Sciences (CAMS-2015), Singapore, pp. 18-21.

Caswell, H. (2001). Matrix Population Models: Construction, Analysis and Interpretation, Sinauer, Sunderland, MA.

Chou, I. and Voit, E. (2013). Recent developments in parameter estimation and structure identification of biochemical and genomic systems, Mathematical Biosciences 219: 57-83.

De La Sen, M. and Quesada, A. (2003). Some equilibrium, stability, instability and ocillatory results for an extended discrete epidemic model with evolution memory, Advances in Difference Equations 2013: 234

Dion, J.M., Commault, C. and van der Woude, J. (2003). Generic properties and control of linear structured systems: A survey, Automatica 39: 1125-1144.

Emmert, H. and Allen, L. (2004). Population persistence and extinction in a discrete-time, stage-structured epidemic model, Journal of Difference Equations and Applications 10(13-15): 1177-1199.

Kaczorek, T. (2002). Positive $1 D$ and 2D Systems, Springer-Verlag, London.

Kajin, M., Almeida, P., Vieira, M. and Cerqueira, R. (2012). The state of the art of population projection models: From the Leslie matrix to evolutionary demography, Oecologia Australis 16(3): 13-22.

Lefkovitch, L. (1965). The study of population growth in organisms grouped by stages, Biometrika 21(1): 1-18.

Leslie, P.H. (1948). Some further notes on the use of matrices in population mathematics, Biometrika 35(3-4): 213-245.

Li, C. and Schneider, H. (2002). Applications of Perron-Frobenius theory to population dynamics, Journal Mathematical Biology 44(5): 450-462.

Li, X. and Wang, W. (2006). A discrete epidemic model with stage structure, Chaos Solitons and Fractals 26(3): 947-958.

Ljung, L. and Soderstrom, T. (1983). Theory and Practice of Recursive Identification, MIT Press, Cambridge, MA

Verdière, N., Denis-Vidal, L., Joly-Blanchard, G. and Domurado, D. (2005). Identifiability and estimation of pharmacokinetic parameters for the ligands of the macrophage mannose receptor, International Journal Applied Mathematics and Computer Science 15(4): 517-526.

Carmen Coll obtained her PhD degree at the Department of Applied Mathematics of the University of Valencia, Spain. At present she is a full professor at the Engineering School of the Polytechnic University of Valencia. She is a member of the Department of Mathematics and the Institute for Multidisciplinary Mathematics of that university. She has participated in the development of several research projects. She has published a number of articles in international journals and with leading conferences. Her fields of interest include applied mathematics, systems theory, especially singular multidimensional systems and positive multidimensional systems, mathematical modelling and identification. Her research is currently focused on applications of mathematical modeling in medicine. 
Elena Sánchez received her $\mathrm{PhD}$ in applied mathematics from the University of Valencia, Spain. Currently she is a professor of the Department of Applied Mathematics and a member of the Institute for Multidisciplinary Mathematics, Polytechnic University of Valencia (UPV). Her main areas of research are matrix analysis control systems theory. She works on linear systems, systems with periodic coefficients and nonnegative systems and their applications to engineering, chemistry and biology. She has published several papers in journals and international conferences, and has participated in several Spanish projects related to these topics.

Received: 9 July 2018

Revised: 17 October 2018

Re-revised: 19 November 2018

Accepted: 29 November 2018 